\title{
BMJ Open Prostate care and prostate cancer from the perspectives of undiagnosed men: a systematic review of qualitative research
}

\author{
Ashwini Kannan, ${ }^{1}$ Maggie Kirkman, ${ }^{2}$ Rasa Ruseckaite, ${ }^{1}$ Sue M Evans ${ }^{1}$
}

To cite: Kannan A, Kirkman M, Ruseckaite R, et al. Prostate care and prostate cancer from the perspectives of undiagnosed men: a systematic review of qualitative research. BMJ Open 2019;9:e022842. doi:10.1136/ bmjopen-2018-022842

- Prepublication history and additional material for this paper are available online. To view these files, please visit the journal online (http://dx.doi. org/10.1136/bmjopen-2018022842).

Received 12 March 2018 Revised 17 October 2018 Accepted 6 November 2018

Check for updates

(c) Author(s) (or their employer(s)) 2019. Re-use permitted under CC BY-NC. No commercial re-use. See rights and permissions. Published by BMJ.

${ }^{1}$ Clinical Registry Unit, Department of Epidemiology and Preventive Medicine, Monash University Australia, Melbourne, Victoria, Australia

${ }^{2}$ School of Public Health and Preventive Medicine, Monash University Australia, Melbourne, Victoria, Australia

Correspondence to

Dr Sue M Evans;

sue.evans@monash.edu

\section{ABSTRACT}

Objectives To summarise and evaluate evidence from men who had not been diagnosed with prostate cancer about their perspectives on prostate care and prostate cancer.

Design A systematic review of qualitative research, on the perspectives of non-cancerous men regarding prostate cancer prevention and care.

Setting A wide range of settings including primary and secondary care.

Participants Men from varied demographic backgrounds ranging between 40 to 80 years of age.

Data sources Three databases (Ovid MEDLINE, Informit, Psychlnfo) and Google Scholar were searched for peerreviewed papers in English reporting research using qualitative methods (in-depth or semistructured interviews and focus groups).

Review methods Thematic analysis using inductive and deductive codes. Thematic synthesis was achieved through iterative open, axial and thematic coding.

Results Eight papers (reporting seven studies conducted in Australia, UK and Germany) met inclusion criteria. Four major themes were identified: understanding prostate cancer, masculinity and prostate cancer, barriers to prostate healthcare and managing prostate health. It was reported that men often did not understand screening, prostate anatomy or their prostate cancer risk, and that concerns about masculinity could deter men from seeking health checks. There was evidence of a need to improve doctor-patient communication about case finding.

Conclusion Further investigation is required to identify and understand any differences in the perspectives and experiences of men who have not been diagnosed with prostate cancer in metropolitan and regional areas, especially where there may be variations in access to healthcare

\section{INTRODUCTION}

Prostate cancer is the second most common male cancer. ${ }^{1}$ It is estimated that, by 2030 , 1.7 million men will be living with prostate cancer. $^{2}$ Australia has the highest incidence of prostate cancer in the world, ${ }^{1}$ attributed largely to a high rate of case-finding, in the form of measuring the concentration of prostate-specific antigen (PSA) in asymptomatic men. An elevated PSA can suggest a tumour of the prostate but it can also signal benign conditions such as infection or prostatic enlargement.

\section{Strengths and limitations of this study}

This review updates previous reviews of men's perspectives on prostate care and prostate cancer, strengthening comparability by including the criterion of research conducted in men within similar healthcare systems.

- The focus on undiagnosed men informs policy and programmes towards preventative strategies in prostate cancer.

- Rigorous and systematic thematic analysis of results produced comprehensive themes.

- The reviewed research included participant diversity in socioeconomic status and, to some extent, ethnicity, although no eligible research on non-heterosexual men was identified.

- The two included papers of only average methodological quality yielded results consistent with remaining papers.

PSA screening is controversial because of the high rate of false positives and the radical treatment given to men with indolent malignancies who would probably die from other causes. ${ }^{3} 4$ Against this is evidence that early detection of prostate cancer and appropriate management can allow for treatment to be initiated at a stage which optimises quality of life while minimising intervention. ${ }^{5}$ There is conflicting evidence of the impact of PSA screening on mortality. ${ }^{6}$

There is consensus that a decision to have a PSA test should be made by patients in consultation with their General $P$ ractitioner (GP) who can explain the risks and benefits of the test as well as risk factors and symptoms of prostate cancer. $^{7}$

It has been found that men are hesitant to visit health professionals ${ }^{8}$; men's underutilisation of the healthcare system constitutes a social problem. ${ }^{9}$ Research has tended to focus on men diagnosed with prostate cancer, with limited evaluation of the perspectives of men who have not been diagnosed. These men may give insight into what leads them to seek or avoid prostate testing and reveal their constructions of prostate cancer in the absence of a 
diagnosis. The aim of this review was to summarise and evaluate evidence from men who had not been diagnosed with prostate cancer about their perspectives on prostate care and prostate cancer.

\section{METHOD}

\section{Search strategy and selection of papers}

The comprehensive search strategy sought all eligible qualitative studies identified using pre-determined Medical Subject Headings (MeSH). Three databases (Ovid MEDLINE, Informit, PsychInfo) and Google Scholar were individually searched with the MeSH: Prostatic Neoplasms, Prostate Cancer, Qualitative Research, Interviews, Attitudes or Behaviours, Health Education and Health Promotion. $\mathrm{MeSH}$ in each relevant article title that was obtained from the first search were screened to ensure that all possible keywords had been considered. Reference lists in each eligible paper were searched for any further eligible articles. Preferred Reporting Items for Systematic Reviews and Meta-Analyses guidelines were used to identify eligible papers. ${ }^{10}$ Identified records were screened by title, then abstract to determine eligibility. Duplicates were removed and the remaining papers were read in full. The search was conducted by AK in consultation with all authors; consensus was reached on eligible papers after individual assessment. This review is reported in accordance with the Enhancing transparency in reporting the synthesis of qualitative research (ENTREQ) guidelines for reviews of qualitative syntheses. ${ }^{11}$

\section{Inclusion criteria}

The most appropriate means of seeking participants' perspectives is to use qualitative methods, including interviews and small group discussions. Papers were therefore eligible for inclusion if they had used qualitative research methods to learn about the perspectives of men who had not been diagnosed with prostate cancer. Papers were not ineligible if they also included men who had been diagnosed with prostate cancer. Where research included men who had been diagnosed with prostate cancer, only results pertaining to undiagnosed men were considered for this review. Other inclusion criteria were that publication was in English in a peer-reviewed journal from 1 January 2000 to 17 October 2018, and that the research had been conducted in Australia or a country with a similar healthcare system (Canada, most of Europe) to limit systemic variation in healthcare available to men (example of search provided in online supplementary text 1 ). Only articles published after 2000 were included to coincide with widespread availability of PSA testing in countries included in this review.

\section{Quality assessment}

The quality of research using qualitative methods cannot be assessed with tools appropriate to quantitative methods because qualitative research seeks complexity, diversity and change, not replicability. ${ }^{12}$ The quality of eligible papers was assessed using the Standard Quality Assessment Criteria for Evaluating Primary Research Papers from a Variety of Fields ${ }^{13}$ with an additional criterion: a statement of approval from an institutional human research ethics committee. Each paper could achieve a maximum score of 22: on each criterion, two points were awarded for yes, one for partial and zero for no. Final scores were defined as strong (17-22 points), adequate (11-16 points) and weak ( $0-10$ points). The appraisal process was conducted by two independent reviewers and any discrepancies were resolved by a third author.

\section{Analysis}

Thematic analysis was conducted on the results presented in each paper, whether in a results section or a results and discussion section. Where results were also presented from men with a prostate cancer diagnosis, we analysed only results pertaining to undiagnosed men. We used a well-established iterative method $^{14}$ consisting of six basic steps. Papers found to be eligible were read in full; themes identified in the results were noted. The themes were systematically assessed and, with the aid of tables and concept maps, organised hierarchically. All aspects of the thematic hierarchy were inspected against the data, involving multiple readings of each paper. The hierarchy was reorganised and the thematic scheme refined to ensure that the themes were comprehensive and appropriate. The analysis was data-driven and thus predominantly inductive. Finally, exemplary quotations were selected. The analysis and its interpretation were repeatedly discussed among all researchers until consensus was reached. As the researcher is the analysis tool in qualitative research, themes will inevitably be tailored to the author's interpretation. However, in this research using qualitative methods, sufficient evidence (eg, quotations) was presented to allow readers to assess the validity of categorisations and interpretations.

\section{Patient and public involvement}

No patients from the primary studies included were involved in this review.

\section{RESULTS}

\section{Study selection}

The initial yield of 767 papers was reduced to eight papers (representing seven studies) in a process summarised in figure 1.

The research was conducted in Australia (four papers/ three studies), the $\mathrm{UK}^{3}$ and Germany. ${ }^{1}$ The number of participants ranged from 20 to 137 men who were aged $40-85$ years; they were commonly recruited from primary care practices. Most of the men were reported to have female partners; no paper reported the inclusion of men with male partners. A summary of the reviewed papers is provided in table 1 .

\section{Quality assessment}

The assessed quality of each paper is presented in table 2. Six papers scored in the strong category, two papers scored average and no paper scored weak. 


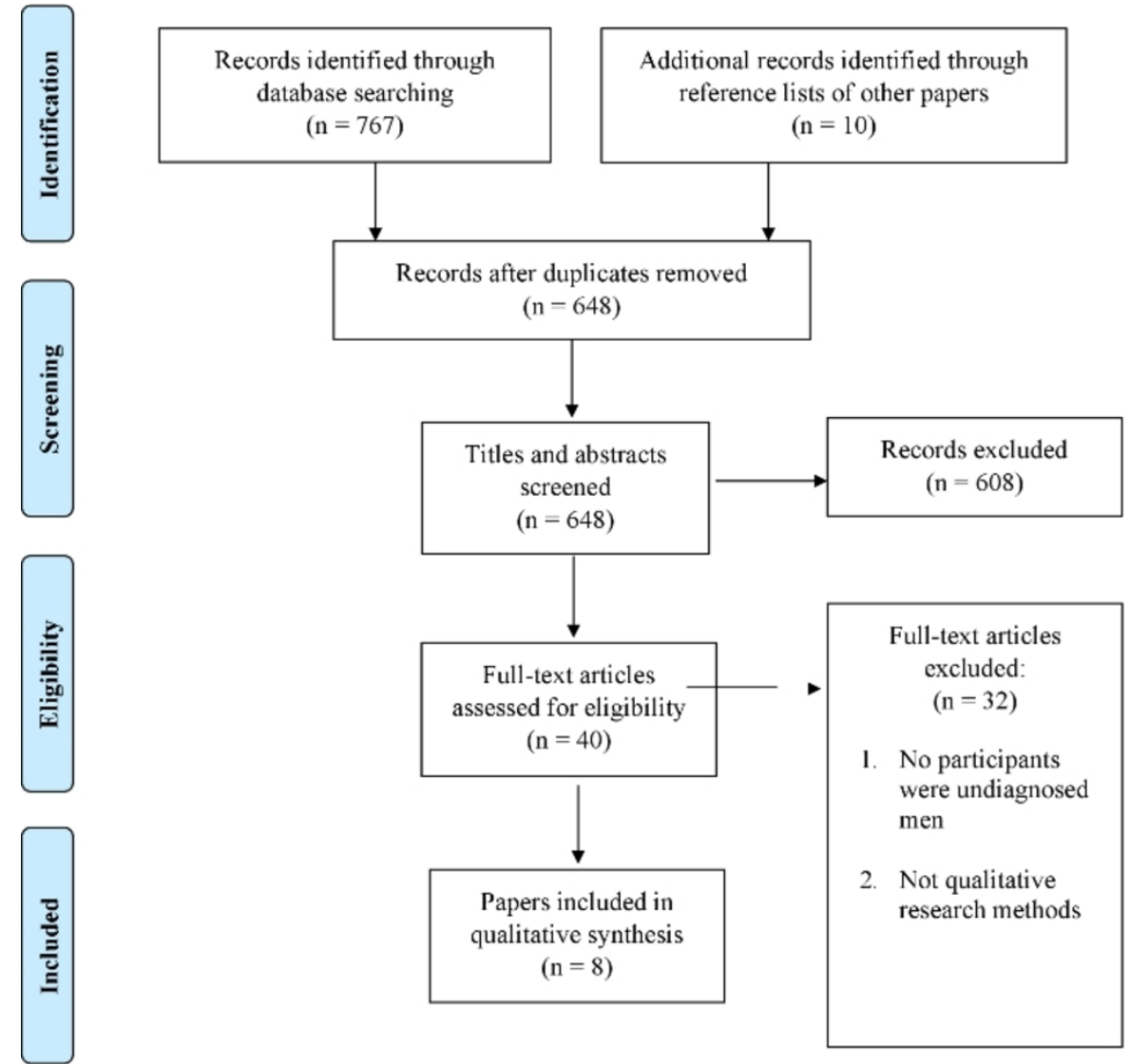

Figure 1 Preferred Reporting Items for Systematic Reviews and Meta-Analyses search strategy and results.

\section{Themes}

Four primary themes were identified: (1) understanding prostate cancer, (2) masculinity and prostate cancer, (3) barriers to prostate care and (4) managing prostate health. It will be evident that the themes are not mutually exclusive.

\section{Understanding prostate cancer}

Researchers reported on men's understanding of the causes and risks of prostate cancer, its signs and symptoms, and its anatomical correlates. In some cases, men's understanding was linked to the likelihood that they would seek testing for prostate cancer, with or without symptoms.

\section{Causes and susceptibility}

Five papers reported men's perceptions of the causes of prostate cancer. ${ }^{15-19}$ Prostate cancer was seen as a common terminal disease caused by ageing; men likened it to a machine wearing out with use. ${ }^{1720}$ Some men also named diet, environment and genetics as causes ${ }^{20}$; others identified healthy behaviour as reducing the likelihood of developing cancer. ${ }^{20}$

Rather than basing the risk of diagnosis on personal factors, men tended to equate their risk to the general population of men their age. ${ }^{17}$ Some men with a family history of cancer did not feel that they were at extra risk. ${ }^{15}$ Horwood $e t a l^{20}$ found that men believed that dietary interventions (green tea and lycopene) reduced risk. ${ }^{20}$
While Bancroft et al and Horwood et $a l^{20}$ exclusively recruited men with a family history of prostate cancer, only Bancroft et al discussed genetic risk in detail. ${ }^{20}{ }^{21}$ They found that being informed of the level of risk did give men a sense of comfort which, however, was limited because they intended to have regular PSA tests and felt at higher risk despite low-risk genetic results. Other men felt that such knowledge was irrelevant because genetics could not be controlled, although a few men considered that modification might be possible in future. These men emphasised the importance of genes that indicate earlier onset of potentially metastatic prostate cancer in younger men. Participants urged further research.

Unsurprisingly, Bancroft et al reported that many men did not fully understand the genetic foundation of prostate cancer. ${ }^{21}$ Nevertheless, these men had greater knowledge of the disease than found elsewhere. ${ }^{21}$ This could be partly attributed to the fact that this research was conducted with men who had participated in a germline genetic profiling study and, before being interviewed, had completed a questionnaire about their experience of genetic testing for prostate cancer. (A second paper, Horwood $e t a l^{20}$ reported on a second project conducted with men recruited from the same original study.)

\section{Symptoms}

Four papers reported that men were uncertain about the symptoms of prostate cancer. ${ }^{15161819}$ Uncertainty was 


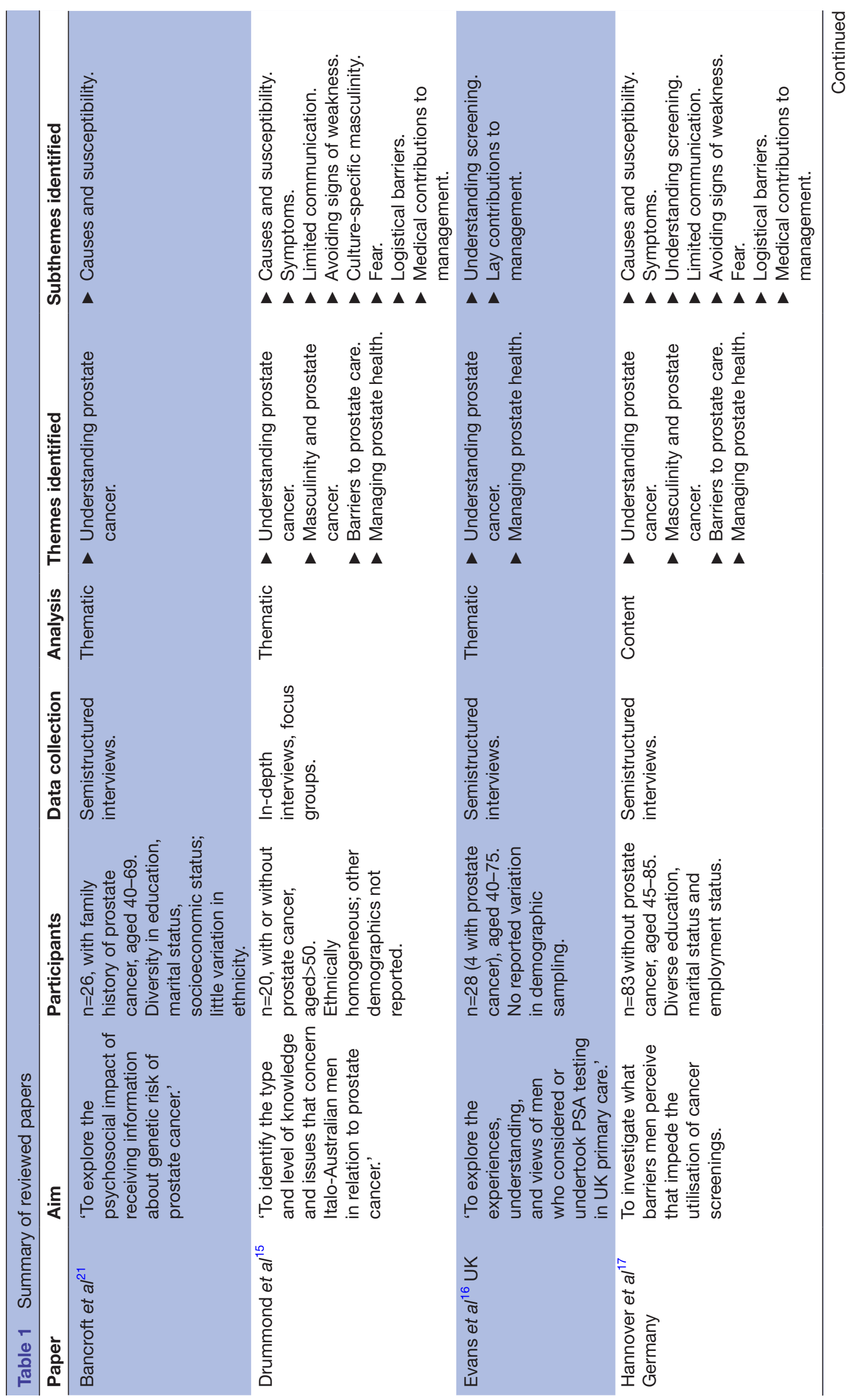




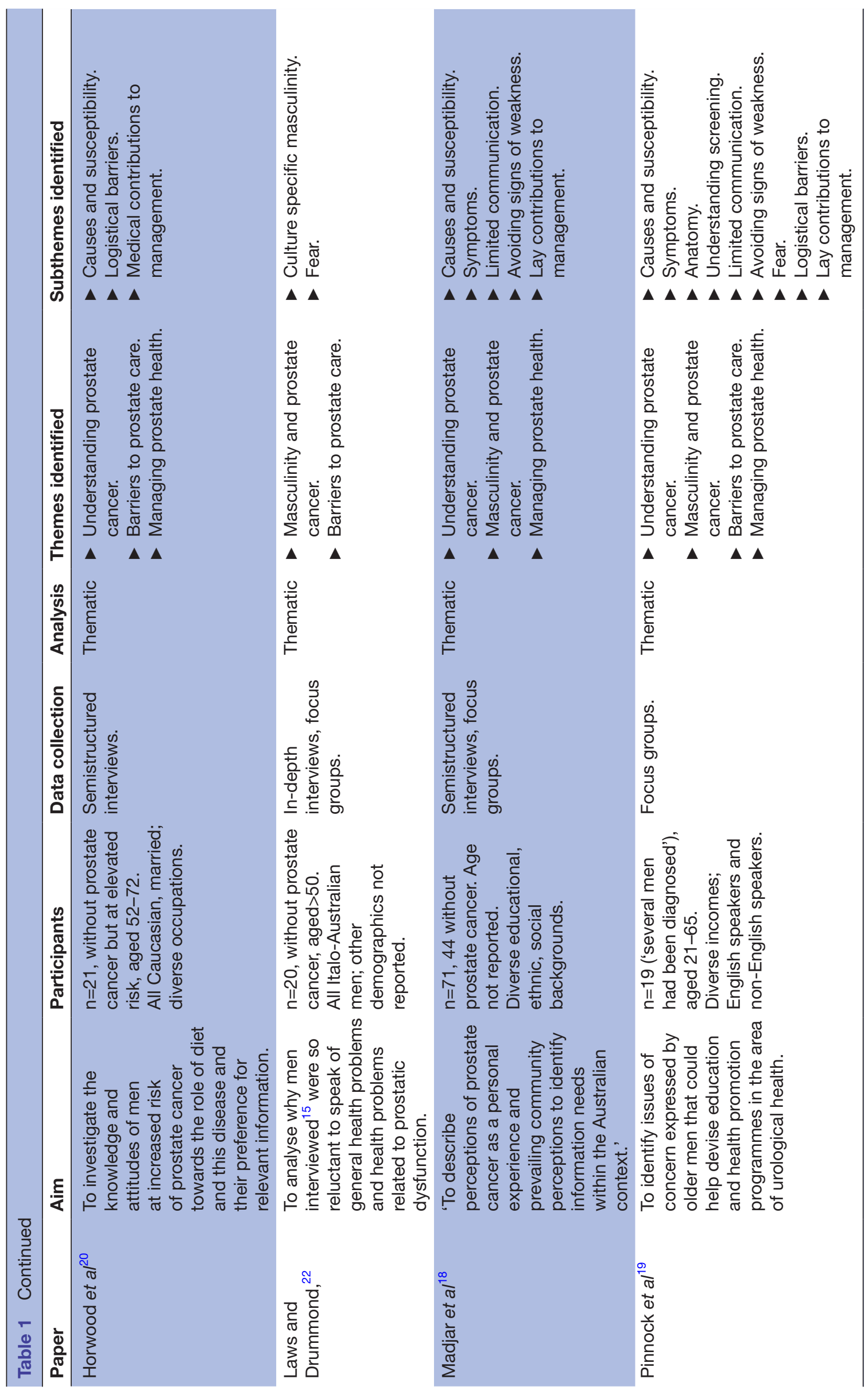




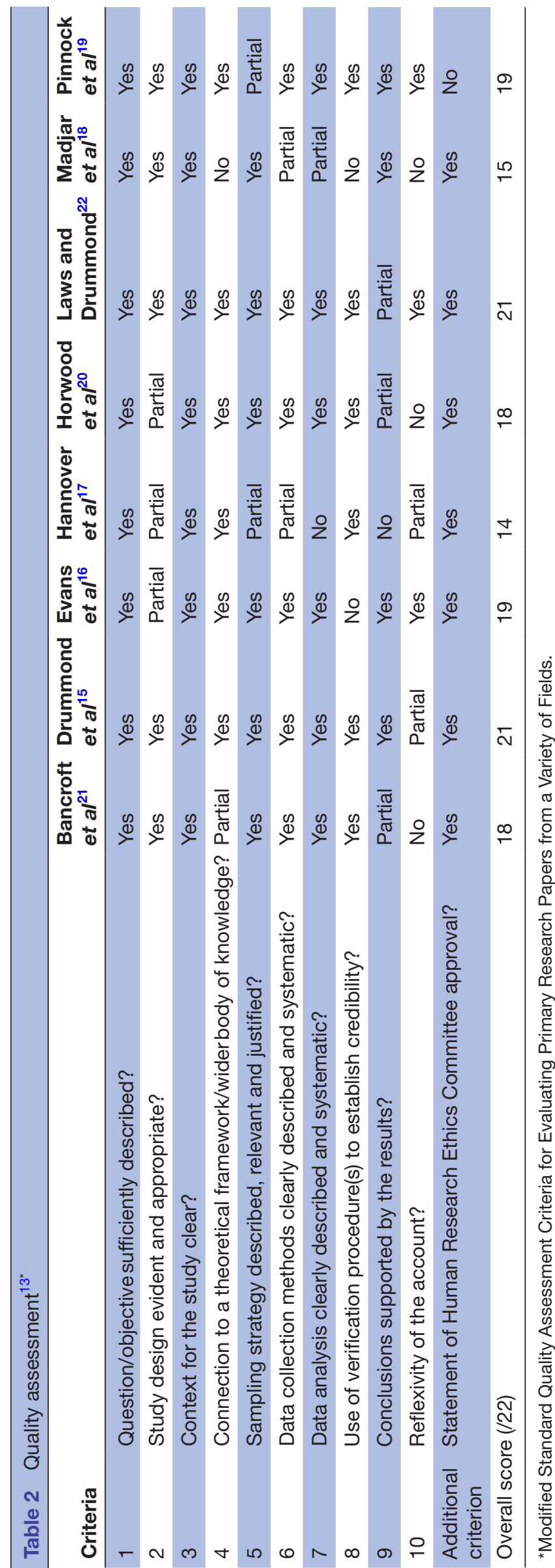

attributed to inadequate information on prostate cancer symptoms and risk factors. ${ }^{16}$ Men used common euphemisms such as 'restriction of waterworks'. ${ }^{15}$ Pinnock et $a l^{19}$ reported that participants did not distinguish symptoms indicative of prostate cancer from signs of ageing. ${ }^{19}$ Although most men could nominate vague urogenital symptoms, investigators found many men to be unaware that prostate cancer was typically asymptomatic. ${ }^{18}$ Men were reported as not being fully aware of the association of incontinence with prostate disease.$^{19}$ However, researchers used the term 'incontinence' loosely, applying it to decreased bladder control and increased frequency of urination (symptomatic of prostate cancer) when it applies strictly to more serious loss of bladder control, a common side-effect of treatment. While Madjar et al acknowledged that the majority of prostate cancer is diagnosed asymptomatically, Pinnock et $a l^{19}$ identified men's limited understanding of symptoms as a concern to be addressed. ${ }^{1819}$

\section{Anatomy}

Men were found in two studies not to recognise the difference among prostate cancer, benign prostatic hyperplasia and other prostatic conditions. ${ }^{18} 19$ Although this might be thought of as expert knowledge, researchers concluded that this confusion arose from men's lack of awareness of prostate anatomy and the aetiology of cancer. ${ }^{15}{ }^{18}$ Men in Madjar et al could not see the need to understand the male reproductive system unless its functionality was threatened. ${ }^{18}$

\section{Understanding screening}

It appeared that men did not fully understand prostate screening. ${ }^{161719}$ They were aware of PSA tests and digital rectal examinations but not of what these tests entailed. ${ }^{17}$ In some cases, men were only vaguely aware of a blood test and were unfamiliar with the abbreviation PSA. ${ }^{16}{ }^{17}$ Men who were credited with comprehension of screening, as some were, identified the poor specificity of PSA tests in differentiating aggressive from benign cancers. ${ }^{16}$

Three papers reported that men lacked knowledge of early detection and its relation to screening. ${ }^{171820}$ Men in these studies thought that prostate cancer would probably develop quite slowly or dissipate without treatment, like a common cold. Other men in the Hannover et al ${ }^{17}$ study reported that they felt early detection was associated with better outcomes and stated their faith in the reliability of medicine.$^{17}$ Although some men in another study seemed to be receptive to screening, they were unsure of how regular testing should be, regardless of the results and were uncertain how to interpret their PSA test results. ${ }^{16}$ Men were more likely to consider being tested if they knew of a friend, relative or celebrity who had done so. ${ }^{19}$ Participants in three studies were not aware of the controversy about screening and regarded limited promotion of PSA testing as a cost-cutting measure by healthcare authorities that prioritised women's health. ${ }^{161719}$ Participants in Pinnock et al expressed interest in improving their knowledge of screening. ${ }^{19}$ 


\section{Masculinity and prostate cancer}

Cultural constructs of masculinity were identified as influencing attitudes to prostate health, evident in limited communication and in concern about being seen as weak. There was evidence of specific cultural forms of masculine expression relevant to prostate cancer.

\section{Limited communication}

It was common for men's reluctance to speak about prostate cancer to be identified. ${ }^{15-19}$ This was attributed by researchers to intergenerational patterns of communication in which fathers perpetuated 'awkward silences' ${ }^{18}$ between themselves and their sons on what were perceived to be sensitive topics, including prostate care. ${ }^{15}$ One research project found evidence of what was described as 'male norms' ${ }^{15}$ of avoidance of communication about health in the rejections reported by some men when they endeavoured to advise their friends to seek help for prostate care. However, these men were willing and able to discuss sensitive topics in a dedicated focus group despite stating that they could not have done so in an ordinary conversation. ${ }^{19}$ Exceptions to the masculine avoidance of certain topics were noted among men who had grown up in a group of peers who faced similar potential ailments; discussion of bodily malfunctions was possible within the group. ${ }^{19}$

\section{Avoiding signs of weakness}

Men were found not to visit a doctor about their prostate unless they were experiencing symptoms such as pain or recurrent nocturnal urination. ${ }^{15} 17$ It was reported in two papers that, if men felt healthy, they thought it was irrelevant to discuss a potential cancer. ${ }^{18} 19$ Men justified their position by claiming that to discuss health concerns or to seek help would be to behave as 'a wuss ${ }^{\text {'18 }}$ and thus inadequately masculine; talking thus about oneself was a sign of weakness and not a 'male thing'. ${ }^{19}$ Embarrassment about the sexual connotations of the prostate also operated as a barrier to recognising symptoms requiring immediate attention and visiting a GP. ${ }^{15} 18$

\section{Culture-specific masculinity}

Two papers from one study reported the perspectives of Italian immigrant men in Australia. ${ }^{15} 22$ It was clear that men who were raised in a particular masculine culture confronted challenges in a new culture that compounded the difficulty of embracing the need for seeking preventive healthcare for the prostate. ${ }^{22}$ Following the emotional and financial struggles of immigration, the imperative to avoid signs of weakness was exacerbated by mistrust of customs and practices in a new country and of information originating from a non-Italian. The researchers concluded that men's tendency to seek the comfort of a familiar culture and a known language perpetuated isolation from the wider community, resulting in limited exposure to the English language and minimal knowledge of contemporary approaches to health. ${ }^{22}$ Immigrants also brought with them ideological and cultural differences from home, such as the antipathy between northern and southern Italians which meant that research participants from northern and middle Italy identified aspects of machismo that would limit southern Italians in managing life and health in a new country. ${ }^{22}$

\section{Barriers to prostate care}

In addition to having limited understanding of prostate cancer, its symptoms and its early detection, and the constraints of prescribed masculinity, fear and logistical problems were identified as barriers to prostate care.

Fear

Fear appeared to be a common thread across papers that reported barriers to prostate care. ${ }^{15} 171922$ Existential fear was a response to the threat of death represented by a diagnosis of prostate cancer, with which was associated words such as death, morbidity and weakness. ${ }^{15} 17$ While nearly half of the men in one study believed that death from undetected prostate cancer was probable, ${ }^{17}$ men in another study were more likely to perceive prostate cancer as non-metastatic and unlikely to be fatal. ${ }^{18}$ While some men acknowledged death as inevitable, others spoke about death with a sense of foreboding and viewed prostate cancer as a death sentence. ${ }^{15} 19$ It was noted that the fatalistic views of men who accepted the ageing process deterred them from adopting healthy diet and exercise; researchers proposed that family and friends were important influences in these attitudes. ${ }^{19}$

Fear of impaired sexual function also acted as a barrier to seeking prostate care. ${ }^{151719}$ This was not applicable to all men, however; some prioritised life over sexual function in older age. ${ }^{19}$

\section{Logistical barriers}

Logistical barriers that discouraged men from seeking assistance for symptoms or having regular health checks were also identified. ${ }^{15} 171920$ The cost of the PSA test, inaccessible clinics, other health problems and lack of time acted as deterrents. ${ }^{17}$ Taking time off work to visit the GP was construed as unfair to co-workers and dependants. ${ }^{17} 19$ The workplace and working conditions could also constitute barriers to care and exacerbate urinary problems: night shifts, inflexible working hours and constant mobility, such as experienced by interstate truck drivers, can reduce accessibility to toilets. ${ }^{19}$

\section{Managing prostate health}

Men's main sources of information about prostate cancer were reported as their families, social networks and GPs. Communication with GPs was identified as important in facilitating health management, but it was evident that men did not always find GPs to provide optimum care.

\section{Lay contributions to management}

Family members, most often wives, could be important in encouraging men to be aware of their health and possible symptoms of disease. ${ }^{161819}$ A change in social networks, such as gaining a new neighbour, could influence men's attitudes to their health. ${ }^{16}$ News and social media also 
played an important role; publicity about celebrities diagnosed with prostate cancer increased awareness. ${ }^{16}$ Italian-Australian men recommended culturally appropriate dissemination of information, such as through Italian newspapers and guest speakers at Italian clubs. ${ }^{15}$

\section{Medical contributions to management}

GPs were identified in four studies as men's primary or most trusted source of health information. ${ }^{15} 171920$ Men were reported as expecting GPs to provide appropriate and authoritative advice and investigation, such as initiating a prostate exam, ${ }^{18}{ }^{19}$ some asserting that taking time to explain information was the hallmark of a good GP. ${ }^{19}$ Men reported great variation in the standard of information imparted doctors. ${ }^{16} 18$

The power imbalance between doctors and their patients was identified by some researchers as the reason men expected doctors, as the experts, to lead discussions of screening ${ }^{1619}$; men saw no reason to question or discuss the doctor's opinion. Men could experience a sense of distrust and confusion with doctors who did not make recommendations about a presenting problem. ${ }^{19}$ Where there was poor communication, men were less likely to make the most beneficial decision. ${ }^{19}$ If patients felt inadequate, perhaps because of language difficulties or limited education, they could accept doctors' decisions without feeling able to ask questions, thus reducing optimum communication. ${ }^{22}$

\section{DISCUSSION}

This systematic review of eight papers (reporting seven studies) that used qualitative methods to examine undiagnosed men's perceptions of and attitudes to prostate cancer has found consistent results that inform understanding of prediagnosis management of prostate care. Men were found to have limited understanding of causes and susceptibility, symptoms, anatomy and processes of screening and case finding. Constructs of masculinity, especially in the perceived prohibition of displaying signs of weakness and resistance to discussing prostate and sexual health, were influential in attitudes to healthcare. Fear and practical difficulties impeded optimum prostate care. Chosen sources of information and encouragement could be lay or medical, with good communication and care from a GP being especially valued.

These results have implications for enhancing enablers and reducing barriers to appropriate prostate care. In particular, GPs have been identified as potentially either contributing to or disrupting men's care, depending on their communication skills. Most men will not have the GP's expert level of knowledge and will need to be appropriately informed and guided. GPs who wait for the patient to raise the topic of prostate care might find that it is never raised or raised too late for effective intervention. For GPs to benefit their patients, they need to be up to date in standards of care and to be given clear guidelines by prostate cancer specialists; it is known that GPs have found conflicting guidelines to be problematic. ${ }^{23}$

Men's partners (wives, in the reviewed research) are clearly valuable enablers of prostate health information-seeking and management; this has been found elsewhere. ${ }^{24}{ }^{25}$ It may thus be important for GPs and policy-makers to continue to be alert to including men's family support systems in early detection and management measures rather than focusing on men themselves.

Evidence from the review reinforces the need to maintain education on prostate health for each generation; it is a continuing task. The reported results, especially but not only in relation to immigrant men, emphasise the necessity to consider appropriate social settings for communicating about prostate care. This could include culture-specific clubs, Men's Sheds and service organisations such as Rotary. The importance of local information dissemination and the use of popular culture to normalise prostate care have been identified (eg, ref 24). The value of peer support is well established. ${ }^{26}{ }^{27}$ Reducing practical barriers to access, such as by making healthcare affordable, assisting workplaces to support men's attendance at clinics and ensuring the availability of accessible care, would also address concerns raised by men in the reviewed research.

A major barrier to prostate (and other health) care is the construct of masculinity that interprets healthseeking as weak and unmanly. The imperative to silent stoicism and resistance to seeking healthcare is a familiar, long-standing construct of masculinity. ${ }^{28} 29$ This presents a complex challenge. However, rapid development in global communication and the ubiquity of social media may offer opportunities to reshape the role of masculine responsibility for health. It has long been known that a gendered approach to health in society is fundamental to public health and individual care. ${ }^{30-32}$

This systematic review of qualitative research concisely summarises what is known about the perspectives of non-cancerous men on prostate care and prostate cancer. Its strength lies in its comprehensive review of eligible peer-reviewed papers and its rigorous analysis of reported results. The consistency of the results generated across the various research projects and from publications since 1998 adds to their validity and suggests potential for wider applicability. A recently published systematic review explored the perceptions of all men, both diagnosed and undiagnosed, on prostate screening. ${ }^{33}$ Despite the discussion of similar themes, this review gives emphasis to a more focused inclusion criteria; men exposed to similar healthcare systems whose opinions were not influenced by personal experiences from an illness trajectory.

There are limitations, all arising from the few papers eligible for review and the inevitable restrictions of scope this entails. Two obvious absences are that papers satisfying the inclusion criteria for this review did not specify having included any non-heterosexual men, and there was no scope for comparing men from metropolitan areas with those from rural and regional areas. The meanings 
of varied access to services and attitudes to healthcare arising from relative population density are therefore not identified in the available data. It is possible that prostate care has different implications for homosexual men, requiring additional sensitivities and support needs; these are also not captured in this review.

\section{CONCLUSION}

Although we have begun to learn from undiagnosed men themselves about what prostate health and prostate cancer mean to them, there is considerable scope for more research to expand knowledge and to inform the continuing improvement in prostate care. Further research could include investigating the effects of having men more involved in decision-making, ways of improving communication with GPs and investigating potential instruments for addressing gendered discourses that discourage help-seeking for prostate care. Results of research on these topics could potentially contribute to enhancing policy and practice.

Acknowledgements The authors acknowledge that Lorena Romero, Librarian of lan Potter Library, Alfred Hospital, contributed substantially to the search strategy of this review.

Contributors Designed the research question and aims: AK, SME, RR, MK. Analysed the data: AK, MK, SME, RR. Wrote the paper: AK, MK. Data interpretation and critical revision of manuscript: AK, SME, RR, MK. All authors reviewed and approved the manuscript.

Funding A Movember Foundation Grant supported this review.

Disclaimer The funders had no role in study conception, design or synthesis.

Competing interests None declared.

Patient consent Not required.

Provenance and peer review Not commissioned; externally peer reviewed. Data sharing statement № additional data are available.

Open access This is an open access article distributed in accordance with the Creative Commons Attribution Non Commercial (CC BY-NC 4.0) license, which permits others to distribute, remix, adapt, build upon this work non-commercially, and license their derivative works on different terms, provided the original work is properly cited, appropriate credit is given, any changes made indicated, and the use is non-commercial. See: http://creativecommons.org/licenses/by-nc/4.0/.

\section{REFERENCES}

1. IARC;WHO. Estimated Age-Standardised rate (world) Incidence and mortality rates: Males. $2012 \mathrm{http} / / / g c o . i a r c . f r / t o d a y / f a c t-s h e e t s$ populations?population $=900 \&$ sex $=1$ (Accessed $10 \mathrm{Apr} 2017$ ).

2. Pakzad R, Mohammadian-Hafshejani A, Ghoncheh M, et al. The incidence and mortality of prostate cancer and its relationship with development in Asia. Prostate Int 2015;3:135-40.

3. Adhyam M, Gupta AK. A review on the clinical utility of psa in cancer prostate. Indian J Surg Oncol 2012;3:120-9.

4. Andriole GL, Crawford ED, Grubb RL, et al. Mortality results from a randomized prostate-cancer screening trial. $N$ Engl J Med 2009;360:1310-9.

5. Dall'Era MA, Albertsen PC, Bangma C, et al. Active surveillance for prostate cancer: a systematic review of the literature. Eur Urol 2012;62:976-83.

6. Fleshner K, Carlsson SV, Roobol MJ. The effect of the USPSTF PSA screening recommendation on prostate cancer incidence patterns in the USA. Nat Rev Urol 2017;14:26-37.
7. Grossman DC, Curry SJ, Owens DK, et al. Screening for prostate cancer: us preventive services task force recommendation statement. JAMA 2018;319:1901-13.

8. Addis ME, Mahalik JR. Men, masculinity, and the contexts of help seeking. Am Psychol 2003;58:5-14.

9. Noone JH, Stephens C. Men, masculine identities, and health care utilisation. Sociol Health IIIn 2008;30:711-25.

10. Moher D, Liberati A, Tetzlaff J, et al. Preferred reporting items for systematic reviews and meta-analyses: the PRISMA statement. PLoS Med 2009;6:e1000097.

11. Tong A, Flemming K, Mclnnes E, et al. Enhancing transparency in reporting the synthesis of qualitative research: ENTREQ. BMC Med Res Methodol 2012;12:181.

12. Liamputtong P. Qualitative research methods, 2009.

13. Kmet LM, Lee RC, Cook LS. Standard quality assessment criteria for evaluating primary research papers from a variety of fields. 13 . Edmonton: Alberta Heritage Foundation for Medical Research (AHFMR) - HTA Initiative \#, 2004.

14. Braun V, Clarke V. Using thematic analysis in psychology. Qual Res Psychol 2006;3:77-101.

15. J. N. Drummond M, A. Laws T, Poljak-Fligic J. Knowledge of and Attitudes towards Prostate Cancer among Italo-Australian Men. Aust J Prim Health 2001;7:9-16.

16. Evans R, Edwards AG, Elwyn G, et al. 'It's a maybe test': men's experiences of prostate specific antigen testing in primary care. $\mathrm{Br}$. J. Gen. Pract 2007;57:303-10.

17. Hannöver W, Köpke D, Hannich HJ. Perceived barriers to prostate cancer screenings among middle-aged men in north-eastern Germany. Public Health Nurs 2010;27:504-12.

18. Madjar I, Denham J, Rashid P. Do women have a role in early detection of prostate cancer? Lessons from a qualitative study. Aust. Fam. Physician 2007;36:375-7.

19. Pinnock C, O'Brien B, Marshall VR. Older men?s concerns about their urological health: a qualitative study. Aust N Z J Public Health 1998;22(3):368-73.

20. Horwood JP, Avery KNL, Metcalfe C, et al. Men's knowledge and attitudes towards dietary prevention of a prostate cancer diagnosis: a qualitative study. BMC Cancer 2014;14:812.

21. Bancroft EK, Castro E, Bancroft GA, et al. The psychological impact of undergoing genetic-risk profiling in men with a family history of prostate cancer. Psychooncology 2015;24:1492-9.

22. Laws TA, Drummond M. The complexities of interviewing ItaloAustralian men about sensitive health issues. Contemp Nurse 2002;12:144-54.

23. Ruseckaite R, Sampurno F, Millar J, et al. Diagnostic and treatment factors associated with poor survival from prostate cancer are differentially distributed between regional and metropolitan Victoria, Australia. BMC Urol 2016;16:54

24. Kirkman M, Young K, Evans S, et al. Men's perceptions of prostate cancer diagnosis and care: insights from qualitative interviews in Victoria, Australia. BMC Cancer 2017;17:704.

25. Hamilton LD, Van Dam D, Wassersug RJ. The perspective of prostate cancer patients and patients' partners on the psychological burden of androgen deprivation and the dyadic adjustment of prostate cancer couples. Psychooncology 2016;25:823-31.

26. Hyde MK, Newton RU, Galvão DA, et al. Men's help-seeking in the first year after diagnosis of localised prostate cancer. Eur J Cancer Care 2017;26:e12497.

27. King AJL, Evans M, Moore THM, et al. Prostate cancer and supportive care: a systematic review and qualitative synthesis of men's experiences and unmet needs. Eur J Cancer Care 2015;24:618-34.

28. Gough B. Try to be healthy, but don't forgo your masculinity: Deconstructing men's health discourse in the media. Soc Sci Med 2006;63:2476-88.

29. Wall DP, Kristjanson LJ, Fisher C, et al. Responding to a diagnosis of localized prostate cancer: men's experiences of normal distress during the first 3 postdiagnostic months. Cancer Nurs 2013;36:44-50.

30. Courtenay WH. Constructions of masculinity and their influence on men's well-being: a theory of gender and health. Soc Sci Med 2000;50:1385-401.

31. Gender CR. health and theory: conceptualizing the issue, in local and world perspective. Soc Sci Med 2012;74:1675-83.

32. Bird CE, Rieker PP. Gender matters: an integrated model for understanding men's and women's health. Soc Sci Med 1999;48:745-55

33. James LJ, Wong G, Craig JC, et al. Men's perspectives of prostate cancer screening: A systematic review of qualitative studies. PLoS One 2017;12:e0188258. 